\title{
Diagnostic Accuracy and Uptake of the PHQ-9 Depression Scale in Pregnancy and the Postpartum Period
}

\author{
Larry Wang ${ }^{1}$, Kurt Kroenke ${ }^{2}$ \\ ${ }^{1}$ Indiana University School of Medicine, ${ }^{2}$ Indiana University School of Medicine, Division of \\ General Internal Medicine and Regenstrief Institute
}

Purpose: Perinatal depression (PND) is a prevalent and disabling problem both during pregnancy and the postpartum period globally. While the legacy screening measure has been the Edinburgh Postnatal Depression Scale (EPDS), the PHQ-9 is the most widely used depression measure in clinical practice and is considered a viable alternative in many PND guidelines. This systematic review examines the criterion and convergent validity of the PHQ-9 as a screener for PND.

Methods: The following databases were searched in June 2020: MEDLINE, Embase, and Psychlnfo. Search terms were the PHQ-9 AND perinatal AND depression. Variants of each were also included as an OR statement such as "patient health questionnaire" as a variant of the PHQ-9. Only studies published in English and after 2001 were accepted as the PHQ-9 was not available as a screening test before then.

Results: A total of 35 articles were eligible for criterion $(n=10)$ or convergent validity $(n=25)$. Studies with criterion validity had sensitivity and specificity ranging from 65 to 95 and 62 to 89 , respectively. For the 7 articles using the standard PHQ-9 cut point $\geq 10$, median sensitivity and specificity were 0.82 and 0.84 , respectively. The median AUC was 0.88 . Of the 9 correlation values shared between the PHQ-9 and EPDS, values range from 0.36 to 0.77 with a median of 0.60. The median Cohen's kappa value of 0.535 in four studies showed good agreement.

Conclusion: Of the criterion validity articles, the sensitivity, specificity, and AUC indicate the PHQ-9 may be used to screen for PND. The convergent validity comparison articles between the PHQ-9 and EPDS also support use of the PHQ-9 in perinatal populations. 